\title{
EL RAZONAMIENTO BIOÉTICO Y LA RESPONSABILIDAD SOCIAL EN EL ALUMNADO DE LAS CARRERAS DEL ÁREA EMPRESARIAL DE LA UNMSM DE PERÚ Y EL CENTRO UNIVERSITARIO LOS LAGOS CULAGOS DE MÉXICO
}

\author{
THE BIOETHICAL REASONING AND SOCIAL RESPONSIBILITY IN THE \\ STUDENTS OF THE RACES OF BUSINESS AREA OF PERU AND UNMSM \\ CENTRAL LAKES COLLEGE OF MEXICO CULAGOS
}

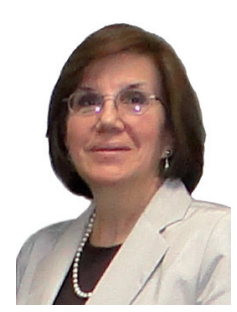

\author{
Ana Maria Gutierrez Huby* \\ Nicko Alberto Gomero Gonzales** \\ Docentes Principales de la Facultad de Ciencias Contables \\ Walter Orlando Tenorio Méndez ${ }^{* * *}$ \\ Docente Asociado de la Facultad de Ciencias Contables \\ Universidad Nacional Mayor de San Marcos-UNMSM / Lima-Perú \\ [Recepción: Julio de 2013/ Conformidad: Agosto 2013]
}

\section{RESUMEN}

Este artículo ha sido elaborado con el objeto de sintetizar los resultados del estudio de investigación Bioética. Componente integral de la responsabilidad social empresarial en los estudiantes de las carreras de contabilidad y administración de la Universidad Nacional Mayor de San Marcos (UNMSM) - Perú y el Centro Universitario de Los Lagos CULAGOS de Guadalajara - México, el cual se elaboró con el interés de indagar en el conocimiento del grado de razonamiento bioético existente en los estudiantes de Pregrado de las carreras de negocio en ambas universidades. Siendo la principal motivación, el buscar sensibilizar a la comunidad universitaria y principalmente, a los conductores de ambos centros, a fin de que inserten la temática como parte de la formación integral de los futuros profesionales del campo empresarial. Los resultados más relevantes son: para los estudiantes de ambos centros, el índice de orientación social empresarial se inclinó hacia el cumplimiento de los aspectos legales y económicos; en lo referente a la consideración de lo que es una empresa exitosa y socialmente responsable, ambos centros consideraron que las características económicas priman para el caso de ser exitosas y para ser socialmente responsables es más importante un comportamiento ético que visualice las necesidades de los grupos de interés.

\section{PALABRAS CLAVE:}

Bioética, razonamiento bioético, responsabilidad social empresarial.

\section{ABSTRACT}

This article has been prepared in order to summarize the results of the research study entitled: Bioethics. Integral component of corporate social responsibility in students majoring in Accounting and Administration careers of the universities of San Marcos, Peru and University Center of Los Lagos CULAGOS of Guadalajara - Mexico.

The study was developed to investigate the interest in understanding the degree of existing bioethical reasoning in Undergraduate students in business careers in both universities. The main objective was to seek motivation in the University Community and especially to the boards of both centers in order to insert the subject as part of the integral education for future professionals in the business field.

The most relevant results were: for students from both schools the corporate social orientation index leaned to the compliance with the legal and economic aspects. In relation to the consideration of what is a successful and socially responsible company, both centers considered that the economic characteristics prevail in the concept of being successful. And finally, as to being socially responsible is more important the ethical behavior displayed towards the needs of stakeholders.

\section{KEYWORDS:}

Bioethics, bioethical reasoning, corporate social responsibility.

* Doctora en Administración-USP. Magister en Gestión Empresarial-UNMSM. Email: anamaria01053@yahoo.es

** Doctor en Ciencias Económicas. Magíster en Contabilidad con mención en banca y finanzas - UNMSM.

Email: nickgo_6@msn.com,gomero_econ@yahoo.es.

*** Economista. Docente Investigador. 


\section{INTRODUCCIÓN}

El impacto de la globalización en el ámbito económico han traído como consecuencia un rumbo de cambios en el comportamiento del mundo empresarial, uno de ellos ha sido la responsabilidad social empresarial, la cual viene constituyéndose como un modelo de gestión para las empresas que orienta la actuación de éstas en beneficio de los denominados grupos de interés (stakeholders), con los que mantienen relación.

A este modelo de gestión, que según lo que nos define Carroll (1979) considera en la actuación de las empresas cuatro grandes dimensiones: la económica, social, legal y ética -las dos últimas condicionando el cabal cumplimiento de las dos primeras- se le trata ahora de vincular con la bioética, otro concepto que viene cobrando fuerza en el escenario social actual, que también tiene sus implicancias en el escenario empresarial por cuanto pone en relieve los conflictos éticos presentes y los que seguirán surgiendo en este campo en particular.

Entre algunos de estos conflictos éticos podemos mencionar la contaminación ambiental, el uso de los transgénicos, el acceso a los servicios, entre otros, los cuales son temas un tanto complejos que involucran afectación o transgresión en uno o más de los principios bioéticos propugnados en esta disciplina, como: la autonomía, la no maleficencia, beneficencia y justicia; temáticas que son acogidas por la bioética a quien le corresponde atender y pronunciarse a fin de aportar con las mejores soluciones a través de los canales normativos y de acciones pertinentes.

En este sentido la relación entre responsabilidad social empresarial y la bioética se produce conjugando la aplicación de los principios éticos con el accionar de la empresa, pero a la vez resulta importante hallar espacios para que la empresa en el contexto de la bioética encuentre en la educación las herramientas suficientes y necesarias que le sirvan de medio para la formación, y transmisión de conocimientos que puedan ser fácilmente aprendidos por los futuros profesionales que se encarguen de la gestión empresarial.
Es así que desde este punto de vista resulta necesaria la consideración del desarrollo de lineamientos pedagógicos y didácticos, así como de metodologías docentes que posibiliten a la bioética ser materia de un consciente y crítico dominio social, por parte de quienes gestionan la educación del país, los que deben asumirla y darle el tratamiento académico que le corresponde, procediendo a considerarla como un elemento básico y fundamental en la formación integral de los educandos contemplándola dentro de la currícula como un saber estructurado, ordenado y adecuado para los alumnos e insertándola adicionalmente como un tema transversal o como un área independiente, de acuerdo a la realidad de cada institución educativa. En el caso de la bioética ésta contiene todas las características para ser considerada como tal.

Por lo expuesto el equipo investigador consideró importante aportar con un primer diagnóstico que es desarrollado con herramientas relevantes a fin de conocer la apreciación del alumnado respecto a estos conceptos.

\section{RESULTADOS DEL ESTUDIO}

Los resultados que se presentan a continuación se basan en la aplicación de una encuesta a estudiantes del Pregrado de las Carreras de Contabilidad y Administración de las Universidades de San Marcos y Guadalajara. El diseño original del cuestionario corresponde a Aupperle y otros (1985), no obstante fue reajustado de acuerdo a la metodología adoptada por el estudio de la Universidad de Puerto Rico de las dras. Silvia López Palau y Beatriz Rivera Cruz.

\section{ÍNDICE DE ORIENTACIÓN HACIA LA RES- PONSABILIDAD SOCIAL EMPRESARIAL DESDE LA PERSPECTIVA DE LOS ESTU- DIANTES}

Para conocer el índice de orientación hacia la responsabilidad social empresarial de los estudiantes de ambos centros se les solicitó a los encuestados indicar cuáles de las premisas presentadas, según su criterio, serían las más importantes para el debido funciona- 
miento de una empresa. Cabe señalar, que detrás de las premisas planteadas se mantuvo una clasificación de las mismas según el modelo multidimensional de empresas definidas por Carroll (1979), y según los principios bioéticos de Beauchamp y Childress.

Como resultado, la Tabla $\mathrm{N}^{\circ} 01$ muestra en ambos casos que los mayores promedios recaen en las dimensiones legal y económica, de ello se infiere que en opinión de los estudiantes, de entre todas las dimensiones que forman parte del índice de orientación social empresarial, lo más importante es cumplir los aspectos legales con que opera la empresa, por las consecuencias que acarrea infringir la ley, lo que podría afectar principalmente la economía y hasta su funcionamiento.

El promedio general de la responsabilidad social empresarial (RSE) obtenido para el caso de Perú fue de $80.13 \%$ y para México fue un poco más alto con un $83.13 \%$.

Ello es un indicativo de que los estudiantes entienden la importancia de las múltiples funciones que tienen las empresas, no siendo únicamente el aspecto económico el que prima en su accionar. 
Tabla No 1

Índice de Orientación hacia la Responsabilidad Social Empresarial (RSE)

\section{UNMSM}

\begin{tabular}{|c|c|c|}
\hline Premisas & $\begin{array}{l}\text { Prome- } \\
\text { dio }\end{array}$ & $\begin{array}{c}\text { Desviación } \\
\text { Estándar }\end{array}$ \\
\hline Dimensión Económica & 81.60 & 16.95 \\
\hline Operar eficientemente & 86.38 & 20.43 \\
\hline Ser competitivas & 85.73 & 22.41 \\
\hline Producir riqueza & 81.54 & 21.89 \\
\hline Ser lucrativas & 79.50 & 22.30 \\
\hline Maximizar la ganancia de los accionistas & 74.86 & 24.70 \\
\hline Dimensión Legal & 83.46 & 18.36 \\
\hline $\begin{array}{l}\text { Cumplir con los contratos contraídos según } \\
\text { las normas legales vigentes }\end{array}$ & 86.07 & 20.02 \\
\hline Cumplir con las leyes ambientales & 84.99 & 23.62 \\
\hline $\begin{array}{l}\text { Contratar y compensar al personal según } \\
\text { la ley }\end{array}$ & 84.95 & 22.12 \\
\hline Pagar impuestos según la ley & 84.43 & 23.45 \\
\hline $\begin{array}{l}\text { Producir bienes y servicios según los están- } \\
\text { dares requeridos por ley }\end{array}$ & 82.70 & 22.44 \\
\hline $\begin{array}{l}\text { Acatar las leyes que regulan la libre com- } \\
\text { petencia }\end{array}$ & 77.78 & 23.81 \\
\hline Dimensión Ética & 79.57 & 16.69 \\
\hline Ser reconocida como una empresa ética & 84.88 & 21.89 \\
\hline $\begin{array}{l}\text { Fomentar conductas éticas entre sus cons- } \\
\text { tituyentes }\end{array}$ & 83.54 & 21.89 \\
\hline $\begin{array}{l}\text { Actuar de acuerdo a las normas éticas de } \\
\text { la sociedad }\end{array}$ & 82.93 & 22.74 \\
\hline $\begin{array}{l}\text { Operar de forma ética sin sacrificar las } \\
\text { metas económicas }\end{array}$ & 76.14 & 26.95 \\
\hline $\begin{array}{l}\text { Operar de forma ética aunque se tengan } \\
\text { que sacrificar las metas económicas }\end{array}$ & 70.36 & 27.58 \\
\hline Dimensión de libre discreción (Social) & 76.04 & 18.82 \\
\hline $\begin{array}{l}\text { Ser reconocida como una empresa social- } \\
\text { mente responsable }\end{array}$ & 85.56 & 21.28 \\
\hline $\begin{array}{l}\text { Mejorar la calidad de vida de su comuni- } \\
\text { dad circundante }\end{array}$ & 78.22 & 23.21 \\
\hline Mejorar la calidad de vida de la sociedad & 78.20 & 24.81 \\
\hline Ayudar a resolver problemas sociales & 74.61 & 24.98 \\
\hline $\begin{array}{l}\text { Destinar parte de las ganancias a activida- } \\
\text { des benéficas }\end{array}$ & 63.58 & 29.52 \\
\hline $\begin{array}{l}\text { Dimensión de Razonamiento Bioético } \\
\text { General }\end{array}$ & 79.11 & 18.13 \\
\hline $\begin{array}{l}\text { Actuar de forma justa en sus operaciones y } \\
\text { actividades }\end{array}$ & 85.95 & 21.26 \\
\hline $\begin{array}{l}\text { Evitar que sus operaciones causen cual- } \\
\text { quier tipo de daños a otros }\end{array}$ & 83.34 & 22.73 \\
\hline $\begin{array}{l}\text { Establecer relaciones con diversas entida- } \\
\text { des basadas en el respeto mutuo }\end{array}$ & 77.13 & 24.67 \\
\hline $\begin{array}{l}\text { Beneficiar con sus operaciones y activida- } \\
\text { des a diversos grupos }\end{array}$ & 69.95 & 25.85 \\
\hline Promedio Índice Orientación Social (RES) & 80.13 & 15.40 \\
\hline
\end{tabular}

Centro Universitario de Los Lagos - CULAGOS

\begin{tabular}{|c|c|c|}
\hline Premisas & $\begin{array}{l}\text { Prome- } \\
\text { dio }\end{array}$ & $\begin{array}{l}\text { Desviación } \\
\text { Estándar }\end{array}$ \\
\hline Dimensión Económica & 84.87 & 23.18 \\
\hline Operar eficientemente & 80.74 & 28.15 \\
\hline Ser competitivas & 86.56 & 23.81 \\
\hline Producir riqueza & 84.17 & 21.32 \\
\hline Ser lucrativas & 91.60 & 14.52 \\
\hline Maximizar la ganancia de los accionistas & 85.86 & 22.92 \\
\hline Dimensión Legal & 84.35 & 22.57 \\
\hline $\begin{array}{l}\text { Cumplir con los contratos contraídos } \\
\text { según las normas legales vigentes }\end{array}$ & 81.69 & 26.78 \\
\hline Cumplir con las leyes ambientales & 90.85 & 20.93 \\
\hline $\begin{array}{l}\text { Contratar y compensar al personal según } \\
\text { la ley }\end{array}$ & 89.24 & 19.21 \\
\hline Pagar impuestos según la ley & 85.06 & 23.20 \\
\hline $\begin{array}{l}\text { Producir bienes y servicios según los están- } \\
\text { dares requeridos por ley }\end{array}$ & 83.98 & 24.84 \\
\hline $\begin{array}{l}\text { Acatar las leyes que regulan la libre com- } \\
\text { petencia }\end{array}$ & 82.77 & 23.88 \\
\hline Dimensión Ética & 76.83 & 27.47 \\
\hline Ser reconocida como una empresa ética & 86.25 & 20.42 \\
\hline $\begin{array}{l}\text { Fomentar conductas éticas entre sus cons- } \\
\text { tituyentes }\end{array}$ & 75.25 & 30.51 \\
\hline $\begin{array}{l}\text { Actuar de acuerdo a las normas éticas de } \\
\text { la sociedad }\end{array}$ & 85.10 & 23.52 \\
\hline $\begin{array}{l}\text { Operar de forma ética sin sacrificar las } \\
\text { metas económicas }\end{array}$ & 90.40 & 17.50 \\
\hline $\begin{array}{l}\text { Operar de forma ética aunque se tengan } \\
\text { que sacrificar las metas económicas }\end{array}$ & 82.22 & 22.46 \\
\hline Dimensión de libre discreción (Social) & 78.96 & 21.48 \\
\hline $\begin{array}{l}\text { Ser reconocida como una empresa social- } \\
\text { mente responsable }\end{array}$ & 72.26 & 33.08 \\
\hline $\begin{array}{l}\text { Mejorar la calidad de vida de su comuni- } \\
\text { dad circundante }\end{array}$ & 84.47 & 22.81 \\
\hline Mejorar la calidad de vida de la sociedad & 82.69 & 20.16 \\
\hline Ayudar a resolver problemas sociales & 92.73 & 14.76 \\
\hline $\begin{array}{l}\text { Destinar parte de las ganancias a activida- } \\
\text { des benéficas }\end{array}$ & 78.48 & 27.48 \\
\hline $\begin{array}{l}\text { Dimensión de Razonamiento Bioético } \\
\text { General }\end{array}$ & 84.15 & 26.16 \\
\hline $\begin{array}{l}\text { Actuar de forma justa en sus operaciones y } \\
\text { actividades }\end{array}$ & 81.98 & 27.09 \\
\hline $\begin{array}{l}\text { Evitar que sus operaciones causen cual- } \\
\text { quier tipo de daños a otros }\end{array}$ & 78.23 & 25.50 \\
\hline $\begin{array}{l}\text { Establecer relaciones con diversas entida- } \\
\text { des basadas en el respeto mutuo }\end{array}$ & 69.56 & 31.16 \\
\hline $\begin{array}{l}\text { Beneficiar con sus operaciones y activida- } \\
\text { des a diversos grupos }\end{array}$ & 69.95 & 25.85 \\
\hline Promedio Índice Orientación Social (RES) & 83.13 & 15.35 \\
\hline
\end{tabular}

Fuente: Encuestas estudiantiles.

Elaboración: Propia. 


\section{EL RAZONAMIENTO BIOÉTICO EN EL ÁMBITO EMPRESARIAL DESDE LA PERCEP- CIÓN DE LOS ESTUDIANTES}

A fin de consolidar la obtención de los resultados sobre el índice de orientación hacia la responsabilidad social empresarial de los estudiantes de ambos centros, se plantearon un conjunto de premisas sobre funciones de los empresarios, solicitándoles indicar cuáles de ellas, según su parecer podían catalogarse como las más importantes en la gestión empresarial.

La Tabla No 02 presenta el promedio obtenido en las premisas relacionadas con las funciones de los directivos. Se observó que en ambos centros el $60 \%$ de las funciones presentadas obtuvieron promedios sobre el $80 \%$. La única premisa de razonamiento bioé- tico incluida es evitar daños a otros con sus acciones u omisiones. No obstante todas las demás premisas resultaron con porcentajes relativamente altos, lo que señala una vez más que el razonamiento bioético tiene cabida en el escenario empresarial.

En conclusión en ambos Centros se coincide en señalar que las funciones más importantes de los directivos son las relacionadas con los aspectos económicos y legales, lo cual concuerda con lo expresado sobre la importancia de las dimensiones teóricas de la empresa.

También hay coincidencia en las tres últimas premisas planteadas consideradas como menos importantes lo que indicaría que los aspectos discrecionales o sociales no son percibidos como prioritarios.

Tabla No 2

Importancia de las Funciones de los Directivos de la Empresa

\begin{tabular}{|c|c|}
\hline \multicolumn{2}{|l|}{ UNMSM } \\
\hline Premisas & Promedio \\
\hline Lograr las metas económicas de la organización & 86.73 \\
\hline Cumplir con todas las leyes y normativas vigentes & 86.58 \\
\hline Tomar decisiones justas & 86.18 \\
\hline Operar de forma ética & 85.75 \\
\hline $\begin{array}{l}\text { Procurar el mejoramiento de los constituyentes de } \\
\text { la empresa }\end{array}$ & 82.32 \\
\hline Evitar daños a otros con sus acciones u omisiones & 81.67 \\
\hline $\begin{array}{l}\text { Defender el derecho de la empresa a elegir y } \\
\text { perseguir sus propios intereses }\end{array}$ & 76.95 \\
\hline $\begin{array}{l}\text { Respetar el derecho de diversos grupos a elegir y } \\
\text { procurar sus intereses }\end{array}$ & 73.04 \\
\hline $\begin{array}{l}\text { Procurar el mejoramiento de grupos externos a la } \\
\text { empresa }\end{array}$ & 72.50 \\
\hline Resolver problemas sociales & 71.00 \\
\hline
\end{tabular}

\begin{tabular}{|l|c|}
\hline \multicolumn{2}{|c|}{ Centro Universitario de Los Lagos - CULAGOS } \\
\hline \multicolumn{1}{|c|}{ Premisas } & Promedio \\
\hline Lograr las metas económicas de la organización & 91.52 \\
\hline Cumplir con todas las leyes y normativas vigentes & 89.48 \\
\hline Tomar decisiones justas & 88.21 \\
\hline Operar de forma ética & 86.79 \\
\hline Evitar daños a otros con sus acciones u omisiones & 85.00 \\
\hline $\begin{array}{l}\text { Defender el derecho de la empresa a elegir y } \\
\text { perseguir sus propios intereses }\end{array}$ & 81.51 \\
\hline $\begin{array}{l}\text { Procurar el mejoramiento de los constituyentes } \\
\text { de la empresa }\end{array}$ & 79.73 \\
\hline $\begin{array}{l}\text { Procurar el mejoramiento de grupos externos a } \\
\text { la empresa }\end{array}$ & 79.27 \\
\hline Resolver problemas sociales & 77.39 \\
\hline $\begin{array}{l}\text { Respetar el derecho de diversos grupos a elegir } \\
\text { y procurar sus intereses }\end{array}$ & 76.45 \\
\hline
\end{tabular}

Fuente: Encuestas estudiantiles.

Elaboración: Propia.

ANÁLISIS COMPARATIVO DE LAS EMPRESAS EXITOSAS Y SOCIALMENTE RESPONSABLES DESDE EL PUNTO DE VISTA ESTUDIANTIL

Seles solicitó a los participantes que de un listado con 15 características, indicaran en términos porcentuales cuáles de estas eran necesarias para catalogar a una empresa como exitosa y como socialmente responsable.

En la UNMSM, lo más importante para que las empresas sean exitosas es que sean competitivas, eficientes, lucrativas y que produzcan riqueza. Todo parece indicar que los participantes establecen el éxito de una empresa en términos económicos.

Las características menos importantes para una empresa exitosa, es que no cause daños y que se autoimponga estándares éticos más allá de lo requerido por ley, en consecuencia, reafirman que el éxito no se 
Ana María Gutiérrez Huby

Nicko Alberto Gomero Gonzales

Walter Orlando Tenorio Méndez

mide por la función social o el comportamiento ético, sino por el cumplimiento de las metas económicas.

Respecto a considerar una empresa como socialmente responsable, de las cuatro premisas con mayor valoración, tres fueron identificadas con carácter social y una referida a comportamiento ético, esto quiere decir, que los participantes entienden la RSE como el comportamiento ético sensible a las necesidades de la sociedad y de los grupos de interés.
Adicionalmente, en el caso peruano, para las premisas de menor valoración: no causar daño a otros y autoimponerse estándares más allá de lo requerido por ley, significa que perciben a las organizaciones socialmente responsables como aquellas sin fines de lucro un tanto incapaces de ser lucrativas o producir riqueza.

En el caso de los estudiantes del Centro Universitario de Los Lagos, lo más importante para que las

Tabla No 3

Resultados - Comparación de Empresas Exitosas y Socialmente Responsables

\begin{tabular}{|c|c|c|c|c|c|c|}
\hline \multicolumn{7}{|c|}{$\begin{array}{c}\text { PERÚ } \\
\text { ¿Cuán necesarias son las siguientes caracteristicas para considerar a una empresa exitosa y } \\
\text { socialmente responsable? }\end{array}$} \\
\hline \multirow[t]{2}{*}{ Premisas } & \multicolumn{2}{|c|}{ Empresa Exitosa } & \multicolumn{2}{|c|}{$\begin{array}{l}\text { Empresa Socialmente } \\
\text { Responsable }\end{array}$} & \multirow[t]{2}{*}{$\begin{array}{l}\text { Diferencia } \\
\text { de Medias }\end{array}$} & \multirow[t]{2}{*}{ Sig. } \\
\hline & Promedio & $\begin{array}{l}\text { Desviación } \\
\text { Estándar }\end{array}$ & Promedio & $\begin{array}{l}\text { Desviación } \\
\text { Estándar }\end{array}$ & & \\
\hline Es competitiva & 88.64 & 18.43 & 75.52 & 25.20 & 13.12 & 0.00 \\
\hline Es eficiente & 88.10 & 18.99 & 79.43 & 23.85 & 8.67 & 0.00 \\
\hline Es lucrativa & 87.05 & 19.30 & 68.38 & 28.49 & 18.67 & 0.00 \\
\hline Produce riqueza & 86.21 & 21.11 & 69.63 & 27.41 & 16.58 & 0.00 \\
\hline Maximiza la ganancia de los accionistas & 85.50 & 20.32 & 67.08 & 28.27 & 18.42 & 0.00 \\
\hline $\begin{array}{l}\text { Contribuye al mejoramiento de sus } \\
\text { constituyentes internos }\end{array}$ & 79.58 & 23.33 & 79.38 & 24.18 & 0.20 & 0.00 \\
\hline Es justa en sus operaciones y actividades & 79.27 & 23.32 & 85.15 & 20.60 & -5.87 & 0.00 \\
\hline Cumple con todas las leyes & 78.83 & 24.54 & 86.67 & 19.17 & -7.84 & 0.00 \\
\hline $\begin{array}{l}\text { Se exige estándares de excelencia más allá } \\
\text { de lo requerido por ley }\end{array}$ & 78.58 & 26.74 & 78.10 & 26.27 & 0.48 & 0.00 \\
\hline $\begin{array}{l}\text { Respeta los derechos de sus constituyentes } \\
\text { internos }\end{array}$ & 77.99 & 23.45 & 80.84 & 25.27 & -2.85 & 0.00 \\
\hline $\begin{array}{l}\text { Respeta los derechos de grupos interesados } \\
\text { externos }\end{array}$ & 75.53 & 25.50 & 82.88 & 23.23 & -7.35 & 0.00 \\
\hline Opera de forma ética & 75.18 & 26.94 & 84.83 & 23.53 & -9.65 & 0.00 \\
\hline $\begin{array}{l}\text { Contribuye al mejoramiento de grupos } \\
\text { externos y de la sociedad }\end{array}$ & 73.84 & 27.88 & 84.83 & 20.92 & -10.99 & 0.00 \\
\hline No le causa daños a otros & 72.16 & 26.71 & 84.26 & 23.25 & -12.10 & 0.00 \\
\hline $\begin{array}{l}\text { Se auto impone estándares éticos más allá } \\
\text { de lo requerido por ley }\end{array}$ & 72.06 & 28.44 & 79.27 & 26.71 & -7.21 & 0.00 \\
\hline
\end{tabular}

Fuente: Encuestas estudiantiles.

Elaboración: Propia.

58/ QVIPURAMAYOC | Vol. 21(40) 2013 
EL RAZONAMIENTO BIOÉTICO Y LA RESPONSABILIDAD SOCIAL EN EL ALUMNADO

DE LAS CARRERAS DEL ÁREA EMPRESARIAL DE LA UNMSM DE PERÚ

Y EL CENTRO UNIVERSITARIO LOS LAGOS CULAGOS DE MÉXICO

empresas sean exitosas es que produzcan riquezas, maximicen las ganancias y respeten los derechos de los grupos interesados externos. Aquí podemos observar, que los participantes además de establecer éxitos en términos económicos contemplan también los aspectos sociales de la empresa. Existe una contradicción en los aspectos menos valorados para que una empresa sea exitosa puesto que consideran que no deben ser lucrativas ni competitivas, lo cual se condice con lo aprobado como aspecto positivo.

En relación a considerar una empresa como socialmente responsable consideran las premisas con mayor valoración el ser competitiva, que contribuya al mejoramiento de sus constituyentes internos y no causar daño a otros. Los participantes entienden la responsabilidad social de las empresas como aquel comportamiento sensible a las necesidades y bienestar de sus grupos de interés.

Tabla No 4

Resultados - Comparación de Empresas Exitosas y Socialmente Responsables

\begin{tabular}{|c|c|c|c|c|c|c|}
\hline \multicolumn{7}{|c|}{$\begin{array}{l}\text { MÉXICO: } \\
\text { ¿Cuán necesarias son las siguientes características para considerar a una empresa exitosa y } \\
\text { socialmente responsable? }\end{array}$} \\
\hline \multirow[t]{2}{*}{ Premisas } & \multicolumn{2}{|c|}{ Empresa Exitosa } & \multicolumn{2}{|c|}{$\begin{array}{l}\text { Empresa Socialmente } \\
\text { Responsable }\end{array}$} & \multirow[t]{2}{*}{$\begin{array}{l}\text { Diferencia } \\
\text { de Medias }\end{array}$} & \multirow[t]{2}{*}{ Sig. } \\
\hline & Promedio & $\begin{array}{l}\text { Desviación } \\
\text { Estándar }\end{array}$ & Promedio & $\begin{array}{l}\text { Desviación } \\
\text { Estándar }\end{array}$ & & \\
\hline Es competitiva & 79.64 & 25.39 & 87.90 & 20.63 & -8.26 & 0.00 \\
\hline Es eficiente & 81.94 & 24.25 & 81.37 & 29.29 & 0.56 & 0.00 \\
\hline Es lucrativa & 79.27 & 28.69 & 85.04 & 25.64 & -5.77 & 0.00 \\
\hline Produce riqueza & 92.78 & 13.02 & 82.34 & 22.60 & 10.44 & 0.07 \\
\hline Maximiza la ganancia de los accionistas & 91.98 & 12.89 & 84.64 & 22.36 & 7.34 & 0.00 \\
\hline $\begin{array}{l}\text { Contribuye al mejoramiento de sus } \\
\text { constituyentes internos }\end{array}$ & 85.51 & 18.16 & 87.62 & 18.16 & -2.11 & 0.00 \\
\hline Es justa en sus operaciones y actividades & 85.87 & 22.75 & 75.81 & 32.31 & 10.06 & 0.00 \\
\hline Cumple con todas las leyes & 80.87 & 19.02 & 83.91 & 20.32 & -3.04 & 0.00 \\
\hline $\begin{array}{l}\text { Se exige estándares de excelencia más allá } \\
\text { de lo requerido por ley }\end{array}$ & 81.70 & 21.44 & 86.85 & 22.39 & -5.15 & 0.34 \\
\hline $\begin{array}{l}\text { Respeta los derechos de sus constituyentes } \\
\text { internos }\end{array}$ & 82.74 & 23.81 & 88.53 & 24.77 & -5.79 & 0.01 \\
\hline $\begin{array}{l}\text { Respeta los derechos de grupos interesados } \\
\text { externos }\end{array}$ & 89.35 & 17.86 & 77.54 & 28.57 & 11.81 & 0.00 \\
\hline Opera de forma ética & 82.81 & 23.49 & 84.89 & 28.30 & -2.07 & 0.00 \\
\hline $\begin{array}{l}\text { Contribuye al mejoramiento de grupos } \\
\text { externos y de la sociedad }\end{array}$ & 83.15 & 25.96 & 85.73 & 25.16 & -2.59 & 0.00 \\
\hline No le causa daños a otros & 82.30 & 24.58 & 86.02 & 20.71 & -3.73 & 0.00 \\
\hline $\begin{array}{l}\text { Se auto impone estándares éticos más allá } \\
\text { de lo requerido por ley }\end{array}$ & 83.04 & 26.54 & 85.04 & 25.32 & -2.00 & 0.00 \\
\hline
\end{tabular}




\section{CONCLUSIONES}

1. La complejidad de los problemas éticos en la actualidad ameritan una revisión multidisciplinaria que provea nuevas perspectivas que favorezcan la formación integral de los estudiantes como futuros directivos y empresarios.

2. El estudio contribuye a la investigación de la bioética ya que resalta la importancia de que forme parte de la currícula de los estudiantes de la carrera de Contabilidad y Administración, en razón de que aún se observa a las organizaciones sólo bajo la perspectiva tradicional económica y no en aspectos intangibles como la bíoética y la responsabilidad social.

3. Todo estudio presenta limitaciones, es posible que las respuestas de los participantes del pregrado no necesariamente reflejen su sentir, porque hay que considerar como punto relevante que los alumnos no están ubicados aún profesionalmente en el campo laboral empresarial.

4. Según la Tabla No 1: Índice de Orientación hacia la Responsabilidad Social Empresarial (RSE) se corroboró en parte la importancia de las cuatro dimensiones del modelo Carrol y su grado relativo de importancia.
5. La dimensión legal y económica, así como las funciones de los directivos de las empresas son importantes según la opinión de los participantes de ambos países.

6. Dada la complejidad de los problemas éticos actuales es necesario continuar multidisciplinariamente estos temas para que provean nuevas y variadas perspectivas que mejoren la formación de los estudiantes como futuros directivos y empresarios.

\section{REFERENCIAS BIBLIOGRÁFICAS:}

1. BUENO, Gustavo (2001). ¿Qué es la bioética? Pentalfa Ediciones. Oviedo.

2. BEAUCHAMP, T.L.; CHILDRESS, J.F. (1999). Principios de Ética Biomédica. Masson, Barcelona.

3. LÓPEZ PALAU, Silvia \& RIVERA CRUZ, Beatriz (2012). El Razonamiento Bioético y la Orientación hacia la Responsabilidad Social Empresarial de los Estudiantes de Negocios Latinoamericanos.

4. LLANO ESCOBAR, Alfonso (2006). Bioética y Educación para el Siglo XXI. Editorial Pontificia Universidad Javeriana. Colombia. 